\title{
Weight changes before and after lurasidone treatment: a real-world analysis using electronic health records
}

\author{
Jonathan M. Meyer ${ }^{1}$, Daisy S. Ng-Mak ${ }^{2^{*}} \mathbb{1}$, Chien-Chia Chuang ${ }^{3}$, Krithika Rajagopalan ${ }^{2}$ and Antony Loebel ${ }^{4}$
}

\begin{abstract}
Background: Severe and persistent mental illnesses, such as schizophrenia and bipolar disorder, are associated with increased risk of obesity compared to the general population. While the association of lurasidone and lower risk of weight gain has been established in short and longer-term clinical trial settings, information about lurasidone's association with weight gain in usual clinical care is limited. This analysis of usual clinical care evaluated weight changes associated with lurasidone treatment in patients with schizophrenia or bipolar disorder.

Methods: A retrospective, longitudinal analysis was conducted using de-identified electronic health records from the Humedica database for patients who initiated lurasidone monotherapy between February 2011 and November 2013. Weight data were analyzed using longitudinal mixed-effects models to estimate the impact of lurasidone on patient weight trajectories over time. Patients' weight data $(\mathrm{kg})$ were tracked for 12-months prior to and up to 12-months following lurasidone initiation. Stratified analyses were conducted based on prior use of second-generation antipsychotics with medium/high risk (clozapine, olanzapine, quetiapine, or risperidone) versus low risk (aripiprazole, ziprasidone, first-generation antipsychotics, or no prior antipsychotics) for weight gain.
\end{abstract}

Results: Among the 439 included patients, the mean age was 42.2 years, and $69.7 \%$ were female. The average duration of lurasidone treatment across all patients was 55.2 days and follow-up duration after the index date was 225.1 days. The estimated impact of lurasidone on weight was $-0.77 \mathrm{~kg}$ at the end of the 1-year follow-up. Patients who had received a prior second-generation antipsychotic with medium/high risk for weight gain were estimated to lose an average of $1.68 \mathrm{~kg}$ at the end of the 1-year follow-up.

Conclusions: Lurasidone was associated with a reduction in weight at 1 year following its initiation in patients with schizophrenia or bipolar disorder. Stratified analyses indicated that weight reduction was more pronounced among patients who had received second-generation antipsychotics associated with a higher risk of weight gain prior to lurasidone treatment. These findings are consistent with the results of prior short- and long-term prospective studies and suggest that lurasidone is associated with low risk for weight gain in patients with schizophrenia or bipolar disorder.

Keywords: Weight change, Mental illness, Electronic health records, Lurasidone, Antipsychotic treatment

\section{Introduction}

Severe and persistent mental illnesses, such as schizophrenia and bipolar disorder, are associated with increased risk of obesity [1] compared to the general

\footnotetext{
*Correspondence: daisy.ng-mak@sunovion.com

2 Sunovion Pharmaceuticals Inc., 84 Waterford Drive, Marlborough, MA 01752, USA

Full list of author information is available at the end of the article
}

population. While over one-third of adults in the United States are obese [body mass index (BMI) $\geq 30 \mathrm{~kg} / \mathrm{m}^{2}$ ], [2, $3]$ the prevalence of obesity or being overweight is estimated at $40-63 \%$ among patients with schizophrenia, [4-6] and 49-68\% among patients with bipolar disorder [7-10]. Evidence suggests that the presence of obesity using BMI, or central adiposity by waist circumference criteria, may negatively impact the disease course in 
schizophrenia and bipolar disorder, and increase risks of cardiovascular disease, diabetes, and stroke in addition to reducing health-related quality of life $[6,11]$.

One of the contributing factors for this higher risk of overweight or obesity in patients with schizophrenia or bipolar disorder may be the use of antipsychotics. While antipsychotics are the current standard pharmacological treatment for severe mental illnesses, as a class they are associated with varying rates of adverse metabolic effects, including a propensity for weight gain [12-15]. Proposed biological mechanisms leading to weight gain in patients treated with antipsychotics include changes in leptin [16] and adiponectin [17]. Among the secondgeneration antipsychotic (SGA) medications with extensive historical data, clozapine, olanzapine, quetiapine, and risperidone are associated with medium and high risk for weight gain, while aripiprazole and ziprasidone have a lower risk $[18,19]$. Lurasidone is an SGA associated with lower risk for inducing weight gain and other cardiometabolic abnormalities in controlled trials and long-term extension studies [15, 20-23]. In addition to the metabolic and social burden of obesity related to SGA use, patients consider the issue of weight gain as the most important treatment concern for the management of bipolar depression [24]. Weight gain is one of the primary adverse effects that lead to treatment non-adherence or discontinuation among schizophrenia patients [25-27]. Non-adherence to psychotropic medications has been found to be associated with consequent relapse and hospitalization rates, resulting in greater healthcare resource use and higher healthcare costs [25, 26, 28, 29].

Given these factors, it is important for clinicians to consider SGAs that are not only efficacious but also provide a lower or more acceptable risk of metabolic disturbance in patients with schizophrenia [30-32]. The value of switching to a lower metabolic risk SGA for patients with high metabolic risk has been reported in other studies [30,33]. Selection of such SGAs may potentially lead to lowered metabolic risk, improved overall healthrelated quality of life, and reduced healthcare costs [34]. While the association of lurasidone and lower risk of weight gain has been established in short and longerterm clinical trial settings, information about lurasidone's association with weight gain in usual clinical care is limited. This study sought to assess the effect of lurasidone treatment on body weight among patients with schizophrenia or bipolar disorder in real-world settings.

\section{Methods}

\section{Study design}

This study was a retrospective, longitudinal analysis of de-identified electronic health records. The health records were from the Humedica NorthStar ${ }^{\mathrm{TM}}$ database from February 1, 2011 to December 31, 2013.

\section{Data source}

The Humedica database contains detailed medical information from integrated claims, prescription, and practice management data on approximately 30 million individuals across 38 states in the US [35]. The de-identified data are representative of the US population in terms of distributions of age, gender, and geographical region. Humedica partners with the nation's leading medical groups, integrated delivery networks, and hospital chains to obtain data from their electronic medical records and information technology systems in real time. The data are then normalized, validated, and aggregated to generate a complete and longitudinal view of patient care [35]. The data used in this study were sourced from the Humedica analytics platform. In addition to the inclusion of standard disease diagnosis and procedure details, the Humedica database includes information of direct relevance to this study, including body weight [kilograms $(\mathrm{kg})]$, body mass index [BMI $\left.\left(\mathrm{kg} / \mathrm{m}^{2}\right)\right]$, and prescription drug information obtained from each provider group's platform. While Humedica standardizes these data to ensure consistency across values (i.e., weight in $\mathrm{kg}$ ), there is no additional logic or algorithm applied. All data used in this study were de-identified and are compliant with the Health Insurance Portability and Accountability Act (HIPAA) of 1996. The study did not require institutional review board approval.

\section{Sample selection}

Patients were included in this study if they met the following criteria: (a) had at least one prescription for lurasidone between February 1, 2011 and November 30, 2013 (first lurasidone prescription date was defined as the index date), (b) did not have prescriptions for other antipsychotics or mood-stabilizing agents at the time of the first prescription for lurasidone, (c) did not have any lurasidone prescriptions during the 12 month preindex period, (d) were aged 18 years or older at index, (e) had $\geq 1$ weight recorded during the 12 month pre-index period and $\geq 1$ weight recorded during the 12 month post-index period, (f) had $\geq 1$ medical encounter in the electronic health record system atleast 12 months prior to the index date, and (g) did not receive other first-generation antipsychotics (FGAs) or SGAs during the 12 month post-index period. Patients were followed for 12 months before the first lurasidone prescription (baseline period) and up to 12 months (minimum 30 days) after the index date (follow-up period). 


\section{Study variables}

Demographic characteristics consisted of age at index date (first lurasidone prescription date), gender, and geographic region (Midwest, Northeast, South, West). Duration of follow-up (in days) was truncated at 365 days after the index date. Clinical characteristics included whether the patient had been diagnosed with a schizophrenia spectrum or bipolar disorder [see Appendix: Table 2 for the International Classification of Diseases, Ninth Revision, Clinical Modification (ICD-9-CM) codes], presence of selected comorbidities (i.e., major depressive disorder, substance abuse, hypertension, hyperlipidemia, diabetes, chronic obstructive pulmonary disease; see Appendix: Table 2), and the Charlson Comorbidity Index score [36]. In addition, prior use of FGAs or SGAs, and prior use of other medications (i.e., antidepressants and diuretics) were included. Presence of medication was identified based on the drug names in the electronic health records (see Appendix: Table 3). Pre-index clinical characteristics also included the most recent BMI measure prior to the index date and BMI classification (i.e., underweight $<18.5 \mathrm{~kg} / \mathrm{m}^{2}$, normal weight $18.5-24.9 \mathrm{~kg} / \mathrm{m}^{2}$, overweight $25.0-29.9 \mathrm{~kg} / \mathrm{m}^{2}$, and obese $\geq 30 \mathrm{~kg} / \mathrm{m}^{2}$ ). Characteristics of lurasidone treatment included the duration of treatment in days (defined as the number of days between the first and last prescription for lurasidone during the follow-up period), and the number of lurasidone prescriptions during the follow-up period.

The primary outcome variable for this study was patient weight $(\mathrm{kg})$ during pre-index and follow-up periods. Weight observations were trimmed such that any records with extreme weight values (i.e., less than $25 \mathrm{~kg}$ or greater than $200 \mathrm{~kg}$ ) were dropped. Patients were only allowed one unique weight observation per day. In cases where a patient had multiple weight measures on the same day, the first recorded weight (based on the timestamp) was selected. For patients with multiple height measures, the modal value was selected; if no modal value existed, the median result was selected as it was less affected by outliers. Height observations were trimmed so that any values less than $120 \mathrm{~cm}$ or greater than $220 \mathrm{~cm}$ were dropped from the analysis. The patient's BMI was calculated using a uniform height measure; therefore, changes in BMI over time reflected changes in weight.

\section{Statistical analyses}

Longitudinal mixed-effects modeling of patient weight trajectories over time was conducted to estimate: (a) patient weight on the index date, (b) the slope of weight change between the beginning and the end of the baseline period (i.e., up until the index date), and (c) the slope of weight change between the beginning and the end of the follow-up period (i.e., a maximum of 365 days after the index date). In addition to the analysis of the overall patient cohort, stratified analyses were conducted on subsets of the overall patient cohort. The first stratified analysis split patients into those who did or did not receive a SGA during the pre-index period. The second split the sample into those who received a SGA with a medium or high risk for weight gain (i.e., clozapine, olanzapine, quetiapine, and risperidone) versus low risk for weight gain (aripiprazole, ziprasidone, first-generation antipsychotics, or no prior antipsychotics).

\section{Results}

Of 3491 patients who received at least one lurasidone prescription, 439 patients initiated lurasidone as monotherapy, continued on lurasidone monotherapy, and had multiple weight measures (Fig. 1). Demographics and patient characteristics during the pre-index period are reported in Table 1 . The mean sample age was 42.2 years, and $69.7 \%$ were female. The mean duration of followup after the index date was 225.1 days. At index, $65.8 \%$ of patients had a diagnosis of schizophrenia spectrum or bipolar disorder, and $34.2 \%$ had other or no diagnoses. Almost half of the patients $(45.8 \%)$ had prior use of a SGA; of these, a total of $27 \%$ of patients had prior use of a SGA associated with medium or high risk for weight gain. First-generation antipsychotics were prescribed to $14.1 \%$ of patients in the year prior to lurasidone initiation. Over half of the patients had prior use of antidepressants. Psychiatric and cardiometabolic comorbidities were commonly observed at index. The mean baseline weight for patients included in this analysis was $93.9 \mathrm{~kg}$, and almost two-thirds of patients were classified as obese according to their BMI at index.

The average duration of lurasidone treatment across all patients was 55.2 days, and the majority of patients (76.1\%) received lurasidone for $<90$ days. On average, patients received two lurasidone prescriptions during the study follow-up period. In the year prior to initiating lurasidone treatment, weight increased by a mean value of $1.64 \mathrm{~kg}$. This trend was reversed after lurasidone initiation (the index date), with patients losing an average of $0.77 \mathrm{~kg}$ (Fig. 2a).

Stratified analyses are shown in Fig. 2b, and c. Similar to the overall findings, increases in weight prior to lurasidone initiation and reduction in weight after lurasidone use were observed in all subgroups. The average weight change after lurasidone treatment in subgroups with or without prior use of second-generation antipsychotics were -1.50 and $-0.29 \mathrm{~kg}$, respectively. The average weight changes after lurasidone treatment in the medium/high weight gain risk antipsychotic subgroups and low weight gain risk antipsychotic subgroups were -1.68 and $-0.62 \mathrm{~kg}$, respectively (Fig. $2 \mathrm{c}$ ). 
Patients with $\geq 1$ prescription for lurasidone between $2 / 1 / 2011$ and $11 / 30 / 2013$

$\mathrm{N}=3,491$ )

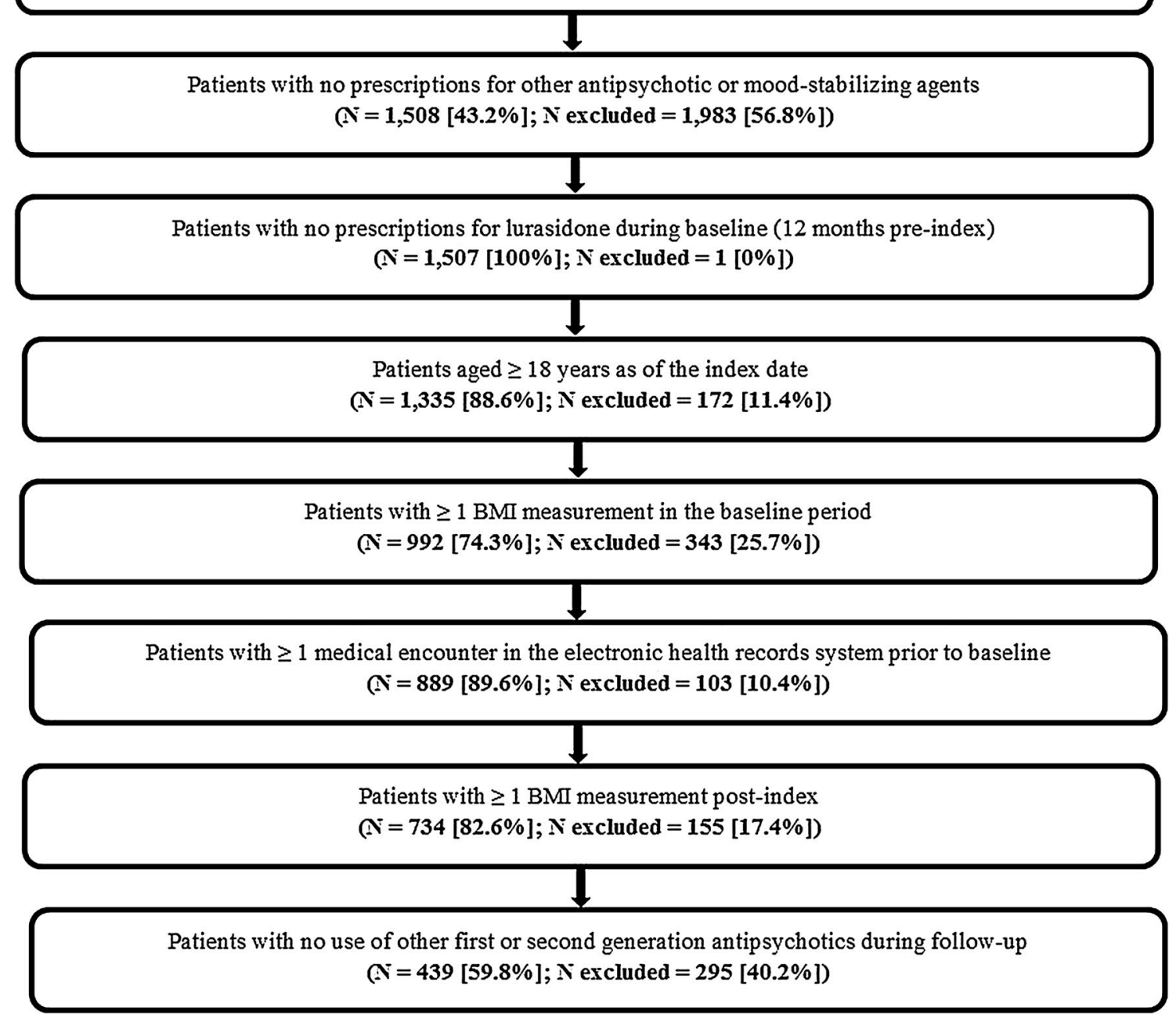

Fig. 1 Patient selection flowchart

\section{Discussion}

This retrospective, longitudinal study using electronic health records from the Humedica database for patients who initiated lurasidone monotherapy between February 2011 and November 2013 examined patterns of weight change before and after initiating lurasidone therapy in patients with schizophrenia or bipolar disorder. Our findings indicated that lurasidone was associated with a reduction in weight, with an estimated average weight loss of $0.77 \mathrm{~kg}$ during the 12 -month follow-up period. Weight reduction associated with lurasidone was more pronounced in a subgroup of patients who had previously received SGAs with medium or high risk for weight gain.

Prior switch trials of antipsychotics among patients who changed from an antipsychotic with a higher weight gain risk to one with lower risk have reported that weight loss occurs gradually following the medication change $[33,37]$. Six-month open-label extension trials of lurasidone have demonstrated that the weight gained during 6 weeks of exposure to olanzapine can be reversed upon a switch to lurasidone, with the weight loss occurring over 3-4 months [38]. A previous analysis evaluated the effect of 12 months' treatment with lurasidone on weight in schizophrenia patients using pooled data from eight clinical trials [13]. Meyer et al. reported that weight loss of at least $7 \%$ was observed in more than twice as many patients receiving lurasidone (18.5\%) than patients receiving risperidone $(6.7 \%)$ or quetiapine extended release (XR) (9.1\%). In addition, patients receiving lurasidone for 12 months were more likely to experience a 
Table 1 Patient demographic and clinical characteristics at index

\begin{tabular}{|c|c|}
\hline Characteristics & Lurasidone $(N=439)$ \\
\hline Age (years), mean (SD) & $42.2(13.5)$ \\
\hline Female, $N(\%)$ & $306(69.7 \%)$ \\
\hline Follow-up duration (days), mean (SD) & $225.1(124.6)$ \\
\hline \multicolumn{2}{|l|}{ Diagnosis of schizophrenia or bipolar disorder, $N(\%)$} \\
\hline Schizophrenia spectrum diagnosis & $83(18.9 \%)$ \\
\hline Bipolar disorder diagnosis & $206(46.9 \%)$ \\
\hline Other/unknown diagnosis ${ }^{a}$ & $150(34.2 \%)$ \\
\hline Prior use of any second-generation antipsychotics $N(\%)$ & $201(45.8 \%)$ \\
\hline Prior use of second-generation antipsychotics associated with medium or higher risk for weight gain ${ }^{b}$ & $119(27.1 \%)$ \\
\hline Prior use of any first-generation antipsychotic, $N(\%)$ & $62(14.1 \%)$ \\
\hline \multicolumn{2}{|l|}{ Prior use of other medications, $N(\%)$} \\
\hline Antidepressants & $246(56.0 \%)$ \\
\hline Diuretics & $90(20.5 \%)$ \\
\hline \multicolumn{2}{|l|}{ Comorbidities, $N(\%)$} \\
\hline Depression & $196(44.6 \%)$ \\
\hline Substance abuse & $166(37.8 \%)$ \\
\hline Hypertension & $158(36.0 \%)$ \\
\hline Hyperlipidemia & $155(35.3 \%)$ \\
\hline Diabetes & $89(20.3 \%)$ \\
\hline Chronic obstructive pulmonary disease & $85(19.4 \%)$ \\
\hline Charlson Comorbidity Index Score, mean (SD) & $0.8(1.3)$ \\
\hline \multicolumn{2}{|l|}{ Weight $^{c}$ and BMI classification ${ }^{d}$} \\
\hline Weight (kg), mean (SD) & $93.9(25.4)$ \\
\hline Underweight, N (\%) & $4(0.9 \%)$ \\
\hline Normal, N (\%) & $70(15.9 \%)$ \\
\hline Overweight, $N(\%)$ & $89(20.3 \%)$ \\
\hline Obese, $N(\%)$ & $276(62.9 \%)$ \\
\hline \multicolumn{2}{|c|}{$\begin{array}{l}\text { As lurasidone would not be prescribed for any other condition except schizophrenia and bipolar, and the lack of a diagnosis in administrative data does not indicate } \\
\text { the lack of the disorder, the data from these patients was deemed acceptable to use in the analysis }\end{array}$} \\
\hline \multicolumn{2}{|c|}{ b Second-generation antipsychotics associated with medium or higher risk of weight gain included clozapine, olanzapine, quetiapine, and risperidone } \\
\hline \multicolumn{2}{|c|}{ c Weight was the most recent weight observation at index (i.e., weight observation closest to and before index date) } \\
\hline \multicolumn{2}{|c|}{$\begin{array}{l}{ }^{d} \text { BMI classification based on the closest BMI reading prior to the index date. Underweight, } \mathrm{BMI}<18.5 \mathrm{~kg} / \mathrm{m}^{2} ; \text { normal weight, } \mathrm{BMI} \geq 18.5 \text { and }<25.0 \mathrm{~kg} / \mathrm{m}^{2} ; \\
\text { overweight, } \mathrm{BMI} \geq 25.0 \text { and }<30 \mathrm{~kg} / \mathrm{m}^{2} ; \text { obese, } \mathrm{BMI} \geq 30 \mathrm{~kg} / \mathrm{m}^{2}\end{array}$} \\
\hline
\end{tabular}

shift from a higher BMI category to a lower BMI category compared with patients treated with risperidone or quetiapine XR [13]. The results of the current study confirm and extend these prior findings by demonstrating weight loss in real-world patients with schizophrenia or bipolar disorder who were switched to lurasidone for up to 12 months.

Patients with serious mental illness have a greater risk for obesity and associated cardiovascular and metabolic diseases [6,39]. Antipsychotic-induced weight gain may exacerbate these conditions and lead to treatment discontinuations $[25,26]$. The results of this study suggest that a potential strategy to manage antipsychotic weight gain may be to switch patients to an antipsychotic with a lower risk of weight gain, such as lurasidone. In this electronic medical record analysis, patients previously treated with SGAs associated with medium/high risk for weight gain had an average weight loss of $1.68 \mathrm{~kg}$ within the 12 months following initiation of lurasidone. Similarly, in a lurasidone switch trial for patients with schizophrenia or schizoaffective disorder, patients lost an average of $0.2 \mathrm{~kg}$ at 6 weeks, and more patients had a clinically significant $(\geq 7 \%)$ weight loss $(1.8 \%)$ than weight gain $(0.9 \%)$ [40]. After 6 months of treatment, the average weight loss was $0.8 \mathrm{~kg}$, with $11.8 \%$ having clinically significant weight loss. Additionally, patients previously treated with a medium/high risk SGA for weight gain [olanzapine (23.1\%) quetiapine (16.7\%), and risperidone $(22.2 \%)$ ] appeared more likely to have a clinically significant weight loss than patients who were previously 

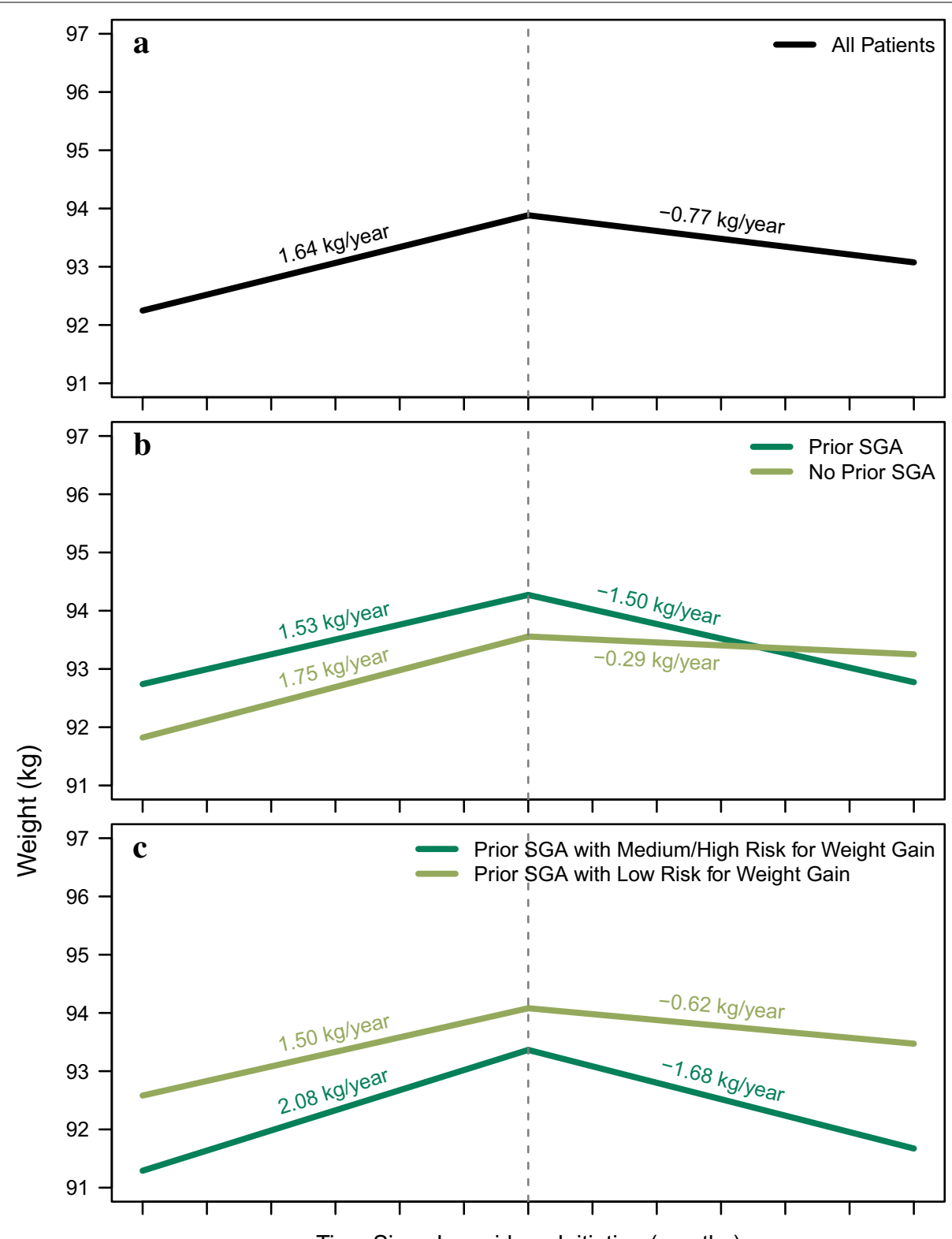

Time Since Lurasidone Initiation (months)

Fig. 2 Impact of lurasidone on weight (a) overall, and stratified by prior use of second-generation antipsychotics (b), and prior use of secondgeneration antipsychotics with medium/high risk for weight gain (c)

treated with a low risk SGA [aripiprazole (3.2\%) or ziprasidone (16.7\%)] [41]. Consistent with these findings, a recent meta-analysis of 15 different antipsychotics in patients with schizophrenia, reported significantly less weight gain in patients treated with lurasidone compared with the majority of treatments [19].

Even a relatively modest degree of weight loss (5\%) has been associated with metabolic benefits [42] and savings in healthcare costs [43]. This suggests that the weight loss observed for lurasidone-treated patients in this study was meaningful from both clinical and cost perspectives.
Pairing the medication switch with a behavioral intervention may lead to an even greater weight loss [44].

The study methodology does have some limitations. The data were collected retrospectively, and other interventions for weight loss could have been implemented by patients at the same time that lurasidone was initiated. Thus, the associations between lurasidone and the reduction in weight following lurasidone initiation could also be due (in whole or in part) to other unobserved interventions such as dietary counseling or prescribed exercise. Mean lurasidone use was only 55.2 days, and 
weight changes associated with lurasidone were estimated at 1 year. The study was a single-group pre-post assessment and did not include a control group. Therefore, no conclusion could be drawn regarding the relative effectiveness of lurasidone for weight management compared to other antipsychotics or add-on treatments like metformin [45]. Only patients with sufficient weight measurements recorded before and after lurasidone initiation were included in this study. Therefore, this study may have been subject to selection bias towards an overweight/ obese cohort whose clinicians were concerned enough to be more assiduous about monitoring weight. It is unclear whether our findings are generalizable to patients with low/normal baseline BMI [46] or to patients who did not gain weight prior to lurasidone treatment. No assessment of cardiometabolic risk factors (e.g., waist circumference or metabolic parameters) were obtained in this study. In addition, assessments of symptomatic change were not available in this study. However, lurasidone is a well-established antipsychotic treatment in patients with schizophrenia and bipolar depression [20, 22-24, 41, 47]. Although one-third of patients in this study did not have a diagnosis code, given the indications for prescribing lurasidone, it is likely that most patients in this study were being treated for schizophrenia spectrum or bipolar disorder.

Another limitation of this study was the linear weight change assumption over a 1-year follow-up period. Prior research suggests that weight change appears to be more pronounced early in treatment and levels off as time passes $[38,48,49]$. To assess the degree to which the choice of a linear model may have resulted in an overestimate of the amount of weight change, a sensitivity analysis (see Additional file 1: Appendix S1) was conducted using 6-month lurasidone data from an open-label extension study [38]. The sensitivity analysis modeled a linear longitudinal mixed-effects model and a nonlinear longitudinal mixed-effects model to test the difference in weight loss estimates. Had a nonlinear model been used in the current study, the weight change estimates may have been about two-thirds the size (see Additional file 1: Appendix S1 for details). While the weight loss estimates may differ somewhat based on whether the weight loss was linear or nonlinear, our study results suggest that lurasidone treatment resulted in statistically significant and clinically meaningful reduction in weight among patients with schizophrenia and bipolar disorder in real-world settings.

\section{Conclusions}

This retrospective, longitudinal study using electronic health records obtained from real-world treatment settings suggests that lurasidone was associated with a reduction in weight at 1 year following its initiation in patients with serious mental illness, primarily schizophrenia or bipolar disorder. Weight reduction was more pronounced among patients who had received secondgeneration antipsychotics associated with a higher risk of weight gain prior to lurasidone treatment. These findings are consistent with the results of prior short- and longterm prospective studies and suggest that lurasidone is associated with low risk for weight gain in patients with schizophrenia or bipolar disorder.

\section{Additional file}

Additional file 1. Analysis examining potential overestimation of weight change by linear model.

\section{Abbreviations}

BMI: body mass index; cm: centimeter; FGA: first-generation antipsychotic; HIPAA: Health Insurance Portability and Accountability Act; ICD-9-CM: International Classification of Diseases, Ninth Revision, Clinical Modification; kg: kilogram; SGA: second-generation antipsychotic; XR: extended release.

\section{Authors' contributions}

All authors were involved in the study conception and design, the drafting of the manuscript, and the critical revisions of the manuscript. All authors read and approved the final manuscript.

\section{Author details}

${ }^{1}$ Department of Psychiatry, University of California, San Diego, California, USA. 2 Sunovion Pharmaceuticals Inc., 84 Waterford Drive, Marlborough, MA 01752, USA. ${ }^{3}$ Vertex Pharmaceuticals, Cambridge, MA, USA. ${ }^{4}$ Sunovion Pharmaceuticals Inc., Fort Lee, NJ, USA.

\section{Acknowledgements}

Medical writing support was provided by Anita Fitzgerald of York Health Economics Consortium Ltd. and Dr. Michael Stensland of Agile Outcomes Research, Inc., and was funded by Sunovion Pharmaceuticals Inc. Statistical analytical support was provided by Dr. Robert Griffiths of Boston Health Economics which was funded by Sunovion Pharmaceuticals Inc.

\section{Competing interests}

Jonathan Meyer, MD, has received research support from Bristol-Myers Squibb, National Institutes of Health (as General Clinical Research Center support), National Institute of Mental Health, and Pfizer Inc., and speaking or advising fees from Acadia Pharmaceuticals, Alkermes, Allergan, Arbor Scientia, BristolMyers Squibb Company, Forum Pharmaceuticals Inc., Genentech, Neuroscience Education Institute, Neurocrine, Inc., Otsuka America Inc., Sunovion Pharmaceuticals Inc., and Teva Pharmaceuticals. Daisy Ng-Mak, Ph.D., Krithika Rajagopalan, Ph.D., and Antony Loebel, MD are employees of Sunovion Pharmaceuticals Inc. Chien-Chia Chuang, Ph.D. was an employee of Sunovion Pharmaceuticals Inc. at the time of the development and write-up of the manuscript and is currently an employee at Vertex Pharmaceuticals.

\section{Availability of data and materials}

The secondary data included in this study is available from Humedica. Restrictions apply to the use of this data, which was used under license for the current study. Study data is available from the authors upon reasonable request and with permission of Humedica.

Consent for publication

Not applicable.

Ethics approval and consent to participate Not applicable. 
Funding

This research was funded by Sunovion Pharmaceuticals Inc., Marlborough, MA, USA.

\section{Previous presentations}

Ng-Mak D, Rajagopalan K, Griffiths R, Lin I, Kanevsky E, Loebel A. Patterns of weight change before and after lurasidone initiation in patients with serious mental illness. Poster presented at: College of Psychiatric and Neurologic Pharmacists (CPNP), April 19-22, 2015, Tampa, FL.

Ng-Mak D, Rajagopalan K, Griffiths R, Lin I, Kanevsky E, Loebel A. Weight changes before and after lurasidone treatment in patients with serious mental illness: real-world analysis using electronic health record (EHR). Poster presented at: Academy of Managed Care Pharmacy (AMCP), April 7-10, 2015, San Diego, CA

\section{Appendix}

See Tables 2, 3.

\section{Table 2 ICD-9-CM codes used to classify mental and somatic disorders}

\begin{tabular}{ll}
\hline Comorbidities & ICD-9-CM codes \\
\hline Schizophrenia & $295 . X X$ \\
Bipolar disorder & $296.01,296.02,296.03,296.04$, \\
& $296.05,296.06,296.40,296.41$, \\
& $296.42,296.43,296.44,296.45$, \\
& $296.46,296.50,296.51,296.52$, \\
& $296.53,296.54,296.55,296.56$, \\
& $296.60,296.61,296.62,296.63$, \\
& $296.64,296.65,296.66,296.7$, \\
Major depressive disorder & $296.2 X, 296.3 X, 311 . X X$ \\
Substance abuse & $296.5 x, 303 . x X-305 . x X$ \\
Hypertension & $401-405$ \\
Hyperlipidemia & $272.2-272.4$ \\
Diabetes & $250 . X X$ \\
Chronic obstructive pulmonary & $490-496,500-505,506.4$ \\
disease &
\end{tabular}

ICD-9-CM The International Classification of Diseases, Ninth Revision, Clinical Modification. ICD-9-CM was the diagnostic coding available in administrative data during the period of this study

\section{Table 3 Medications used in database query}

\begin{tabular}{lc}
\hline Drug name & Drug name \\
\hline First-generation antipsychotics & $\begin{array}{c}\text { Chlorpromazine, fluphenazine, } \\
\text { haloperidol, loxapine, perphena- } \\
\text { zine, pimozide, thiothixene, thiori- } \\
\text { dazine, and trifluoperazine }\end{array}$ \\
Second-generation antipsychotics & $\begin{array}{c}\text { Clozapine, olanzapine, quetiapine, } \\
\text { risperidone, paliperidone, ari- } \\
\text { piprazole, ziprasidone, iloperi- } \\
\text { done, and asenapine }\end{array}$ \\
$\begin{array}{c}\text { Second-generation antipsychotics } \\
\text { that placed patients at higher risk } \\
\text { of weight gain }\end{array}$ & $\begin{array}{c}\text { Clozapine, olanzapine, quetiapine, } \\
\text { risperidone }\end{array}$ \\
Antidepressants & \\
Diuretics & \\
\hline
\end{tabular}

\section{Publisher's Note}

Springer Nature remains neutral with regard to jurisdictional claims in published maps and institutional affiliations.

Received: 7 May 2017 Accepted: 11 October 2017

Published online: 17 October 2017

\section{References}

1. Zhao Z, Okusaga OO, Quevedo J, Soares JC, Teixeira AL. The potential association between obesity and bipolar disorder: a meta-analysis. $J$ Affect Disord. 2016;202:120-3.

2. Flegal KM, Kruszon-Moran D, Carroll MD, Fryar CD, Ogden CL. Trends in obesity among adults in the United States, 2005 to 2014. JAMA. 2016;315:2284-91.

3. Ogden $\mathrm{CL}$, Carroll MD, Fryar CD, Flegal KM. Prevalence of obesity among adults and youth: United States, 2011-2014. NCHS Data Brief. 2015;219:1-8.

4. Catapano L, Castle D. Obesity in schizophrenia: what can be done about it? Australas Psychiatry. 2004;12:23-5.

5. Dickerson FB, Brown CH, Daumit GL, Fang L, Lijuan F, Goldberg RW, et al. Health status of individuals with serious mental illness. Schizophr Bull. 2006:32:584-9.

6. Kolotkin RL, Corey-Lisle PK, Crosby RD, Swanson JM, Tuomari AV, Litalien GJ, et al. Impact of obesity on health-related quality of life in schizophrenia and bipolar disorder. Obesity. 2008;16:749-54.

7. Fagiolini A, Frank E, Houck PR, Mallinger AG, Swartz HA, Buysse DJ, et al. Prevalence of obesity and weight change during treatment in patients with bipolar I disorder. J Clin Psychiatry. 2002;63:528-33.

8. Fagiolini A, Frank E, Scott JA, Turkin S, Kupfer DJ. Metabolic syndrome in bipolar disorder: findings from the Bipolar Disorder Center for Pennsylvanians. Bipolar Disord. 2005;7:424-30.

9. Garcia-Portilla MP, Saiz PA, Benabarre A, Sierra P, Perez J, Rodriguez A, et al. The prevalence of metabolic syndrome in patients with bipolar disorder. J Affect Disord. 2008;106:197-201.

10. McElroy SL, Frye MA, Suppes T, Dhavale D, Keck PE, Leverich GS, et al. Correlates of overweight and obesity in 644 patients with bipolar disorder. J Clin Psychiatry. 2002:63:207-13.

11. Lindenmayer JP, Khan A, Kaushik S, Thanju A, Praveen R, Hoffman L, et al. Relationship between metabolic syndrome and cognition in patients with schizophrenia. Schizophr Res. 2012;142:171-6.

12. American Diabetes Association, American Psychiatric Association, American Association of Clinical Endocrinologists, North American Association for the Study of Obesity. Consensus development conference on antipsychotic drugs and obesity and diabetes. J Clin Psychiatry. 2004:65:267-72

13. Meyer JM, Mao Y, Pikalov A, Cucchiaro J, Loebel A. Weight change during long-term treatment with lurasidone: pooled analysis of studies in patients with schizophrenia. Int Clin Psychopharmacol. 2015;30:342-50.

14. Newcomer JW. Second-generation (atypical) antipsychotics and metabolic effects: a comprehensive literature review. CNS Drugs. 2005:19(Suppl 1):1-93.

15. Newcomer JW. Metabolic considerations in the use of antipsychotic medications: a review of recent evidence. J Clin Psychiatry. 2007;68(Suppl 1):20-7.

16. Potvin S, Zhornitsky S, Stip E. Antipsychotic-induced changes in blood levels of leptin in schizophrenia: a meta-analysis. Can J Psychiatry. 2015:60:S26-34.

17. Bartoli F, Crocamo C, Clerici M, Carrà G. Second-generation antipsychotics and adiponectin levels in schizophrenia: a comparative meta-analysis. Eur Neuropsychopharmacol. 2015;25:1767-74.

18. Muench J, Hamer AM. Adverse effects of antipsychotic medications. Am Fam Physician. 2010:81:617-22.

19. Leucht S, Cipriani A, Spineli L, Mavridis D, Orey D, Richter F, et al. Comparative efficacy and tolerability of 15 antipsychotic drugs in schizophrenia: a multiple-treatments meta-analysis. Lancet. 2013;382:951-62. 
20. Citrome L. Lurasidone for schizophrenia: a review of the efficacy and safety profile for this newly approved second-generation antipsychotic. Int J Clin Pract. 2011;65:189-210.

21. Citrome L. Lurasidone in schizophrenia: new information about dosage and place in therapy. Adv Ther. 2012;29:815-25.

22. De Hert M, Yu W, Detraux J, Sweers K, van Winkel R, Correll CU. Body weight and metabolic adverse effects of asenapine, iloperidone, lurasidone and paliperidone in the treatment of schizophrenia and bipolar disorder. CNS Drugs. 2012;26:733-59.

23. Newcomer J, Tocco M, Pikalov A, Zheng H, Cucchiaro J, Loebel A. Metabolic syndrome in patients with schizophrenia receiving long-term treatment with lurasidone, quetiapine $\mathrm{XR}$, or risperidone. Eur Psychiatry. 2016;33(Suppl 1):107.

24. Ng-Mak DS, Poon JL, Rajagopalan K, Kleinman L, Roberts L, Revicki DA, et al. Qualitative study of patients' preferences for bipolar depression treatment. Value Health. 2015;18:A123.

25. Weiden PJ, Kozma C, Grogg A, Locklear J. Partial compliance and risk of rehospitalization among California Medicaid patients with schizophrenia. Psychiatr Serv. 2004;55:886-91.

26. Weiden PJ, Mackell JA, McDonnell DD. Obesity as a risk factor for antipsychotic noncompliance. Schizophr Res. 2004;66:51-7.

27. Lieberman JA, Stroup TS, McEvoy JP, Swartz MS, Rosenheck RA, Perkins DO, et al. Effectiveness of antipsychotic drugs in patients with chronic schizophrenia. N Engl J Med. 2005;353:1209-23.

28. Knapp M, King D, Pugner K, Lapuerta P. Non-adherence to antipsychotic medication regimens: associations with resource use and costs. $\mathrm{Br} J$ Psychiatry. 2004;184:509-16.

29. Panish J, Karve S, Candrilli SD, Dirani R. Association between adherence to and persistence with atypical antipsychotics and psychiatric relapse among US Medicaid-enrolled patients with schizophrenia. J Pharm Health Serv Res. 2013:4:29-39.

30. Stroup TS, Byerly MJ, Nasrallah HA, Ray N, Khan AY, Lamberti JS, et al. Effects of switching from olanzapine, quetiapine, and risperidone to aripiprazole on 10-year coronary heart disease risk and metabolic syndrome status: results from a randomized controlled trial. Schizophr Res. 2013;146:190-5.

31. Stroup TS, McEvoy JP, Ring KD, Hamer RH, LaVange LM, Swartz MS, et al. A randomized trial examining the effectiveness of switching from olanzapine, quetiapine, or risperidone to aripiprazole to reduce metabolic risk: comparison of antipsychotics for metabolic problems (CAMP). Am J Psychiatry. 2011;168:947-56.

32. Uçok A, Gaebel W. Side effects of atypical antipsychotics: a brief overview. World Psychiatry. 2008;7:58-62.

33. Weiden PJ, Newcomer JW, Loebel AD, Yang R, Lebovitz HE. Long-term changes in weight and plasma lipids during maintenance treatment with ziprasidone. Neuropsychopharmacology. 2008;33:985-94.

34. Van Gaal LF. Long-term health considerations in schizophrenia: metabolic effects and the role of abdominal adiposity. Eur Neuropsychopharmacol. 2006;16(Suppl 3):S142-8.

35. Humedica life sciences: detailed clinical data. Humedica, Boston. 2013. http://www.optum.ca/content/dam/optum/resources/productSheets/ Optum_Humedica_NorthStar_brochure.pdf. Accessed 06 Mar 2017.

36. Deyo RA, Cherkin DC, Ciol MA. Adapting a clinical comorbidity index for use with ICD-9-CM administrative databases. J Clin Epidemiol. 1992:45:613-9.
37. Tarricone I, Ferrari Gozzi B, Serretti A, Grieco D, Berardi D. Weight gain in antipsychotic-naive patients: a review and meta-analysis. Psychol Med. 2010:40:187-200.

38. Stahl SM, Cucchiaro J, Simonelli D, Hsu J, Pikalov A, Loebel A. Effectiveness of lurasidone for patients with schizophrenia following 6 weeks of acute treatment with lurasidone, olanzapine, or placebo: a 6-month, openlabel, extension study. J Clin Psychiatry. 2013;74:507-15.

39. Fagiolini A, Kupfer DJ, Houck PR, Novick DM, Frank E. Obesity as a correlate of outcome in patients with bipolar I disorder. Am J Psychiatry. 2003;160:112-7.

40. McEvoy JP, Citrome L, Hernandez D, Cucchiaro J, Hsu J, Pikalov A, et al. Effectiveness of lurasidone in patients with schizophrenia or schizoaffective disorder switched from other antipsychotics: a randomized, 6-week, open-label study. J Clin Psychiatry. 2013;74:170-9.

41. Citrome L, Weiden PJ, McEvoy JP, Correll CU, Cucchiaro J, Hsu J, et al. Effectiveness of lurasidone in schizophrenia or schizoaffective patients switched from other antipsychotics: a 6-month, open-label, extension study. CNS Spectr. 2014;19:330-9.

42. Blackburn G. Effect of degree of weight loss on health benefits. Obes Res. 1995:3(Suppl 2):211s-6s.

43. Cawley J, Meyerhoefer C, Biener A, Hammer M, Wintfeld N. Savings in medical expenditures associated with reductions in body mass index among US adults with obesity, by diabetes status. Pharmacoeconomics. 2015;33:707-22.

44. Green CA, Yarborough BJH, Leo MC, Yarborough MT, Stumbo SP, Janoff $\mathrm{SL}$, et al. The STRIDE weight loss and lifestyle intervention for individuals taking antipsychotic medications: a randomized trial. Am J Psychiatry. 2015;172:71-81.

45. Newall H, Myles N, Ward PB, Samaras K, Shiers D, Curtis J. Efficacy of metformin for prevention of weight gain in psychiatric populations: a review. Int Clin Psychopharmacol. 2012;27:69-75.

46. Lee CMY, Huxley RR, Wildman RP, Woodward M. Indices of abdominal obesity are better discriminators of cardiovascular risk factors than BMl: a meta-analysis. J Clin Epidemiol. 2008;61:646-53.

47. Franklin R, Zorowitz S, Corse AK, Widge AS, Deckersbach T. Lurasidone for the treatment of bipolar depression: an evidence-based review. Neuropsychiatr Dis Treat. 2015;11:2143-52.

48. Bushe CJ, Slooff CJ, Haddad PM, Karagianis JL. Weight change from 3-year observational data: findings from the worldwide schizophrenia outpatient health outcomes database. J Clin Psychiatry. 2012;73:e749-55.

49. Millen BA, Campbell GM, Beasley CM. Weight changes over time in adults treated with the oral or depot formulations of olanzapine: a pooled analysis of 86 clinical trials. J Psychopharmacol. 2011;25:639-45

\section{Submit your next manuscript to BioMed Central and we will help you at every step:}

- We accept pre-submission inquiries

- Our selector tool helps you to find the most relevant journal

- We provide round the clock customer support

- Convenient online submission

- Thorough peer review

- Inclusion in PubMed and all major indexing services

- Maximum visibility for your research

Submit your manuscript at www.biomedcentral.com/submit 